\title{
Laparoscopic Approach to Barrett Carcinoma in an Obese Patient
}

\author{
Georg Wiltberger, MD, Felix Krenzien, MD, Hans-Michael Hau, MD, Hans-Michael Tautenhahn, \\ Albrecht Hoffmeister, MD, Sven Jonas, MD, Arne Dietrich, MD \\ Department of Visceral, Transplantation, Thoracic and Vascular Surgery, University Hospital Leipzig, Leipzig, Germany \\ (Drs. Wiltberger, Krenzien, Hau, Tautenhahn, Jonas, Dietrich). \\ Department of Medicine, Dermatology and Neurology, Division of Gastroenterology and Rheumatology, University of \\ Leipzig, Leipzig, Germany (Dr. Hoffmeister).
}

\begin{abstract}
We report an obese patient who was initially scheduled for laparoscopic Roux-en-Y gastric bypass, but who was subsequently diagnosed preoperatively with Barrett carcinoma. After incomplete endoscopic mucosectomy, laparoscopic oncologic resection (transhiatal extended cardia resection) combined with bariatric reconstruction was performed. Sufficient complication management of a small anastomotic leak into the right pleural space was maintained with a combination of video-assisted thoracoscopy and endoscopic clipping of the leak.
\end{abstract}

Key Words: Barrett carcinoma, Bariatric surgery, LRYGB, Merendino.

Citation Wiltberger G, Krenzien F, Hau H-M, Tautenhahn H-M, Hoffmeister A, Jonas S, Dietrich A. Laparoscopic approach to barrett carcinoma in an obese patient CRSLS e2014.00132. DOI: 10.4293/CRSLS.2014.00132.

Copyright (C) 2014 SLS This is an open-access article distributed under the terms of the Creative Commons Attribution-Noncommercial-ShareAlike 3.0 Unported license, which permits unrestricted noncommercial use, distribution, and reproduction in any medium, provided the original author and source are credited.

Address correspondence to: Georg Wiltberger, MD, Liebigstraße 20, 04103 Leipzig, Germany, Telephone: (+49) (0) 341-971-7200, Fax: (+49) (0) 341-971-7209, E-mail Georg.Wiltberger@medizin.uni-leipzig.de

\section{INTRODUCTION}

Obesity is a worldwide epidemic associated with an increased risk of type 2 diabetes, cardiovascular disease, gastroesophageal reflux disease (GERD), and cancer. ${ }^{1}$ Bariatric surgery remains the most effective treatment for morbid obesity. Because of its high rates of long-term weight loss and significant reduction in patient comorbidities, laparoscopic Roux-en-Y gastric bypass (LRYGB) is the most widely used procedure. Prior to bariatric surgery, preoperative upper endoscopy should be performed routinely to exclude relevant gastrointestinal (GI) diseases. In early Barrett carcinoma, an endoscopic mucosectomy is recommended, according to recent guidelines. ${ }^{2}$ If histologic analysis reveals an incomplete resection or an early tumor stage, a modified resection of the cardia and lower esophagus as described by Merendino may be performed in selected cases. ${ }^{3}$ This case report shows the feasibility of a modified oncologic resection combined with a bariatric reconstruction and highlights the postoperative complication management.

\section{CASE DESCRIPTION}

A 44-year-old obese man (164 kg, body mass index 45.5 $\mathrm{kg} / \mathrm{m}^{2}$ ) with associated comorbidities (type 2 diabetes mellitus, hypertension, and GERD) attended an interdisciplinary weight loss program for $>6$ months, which included frequent physical exercises, behavioral education, and diet counseling, without achieving significant weight loss. After ruling out endocrinological and psychological causes, the patient was scheduled for LRYGB. A preoperative routinely performed upper endoscopy showed a polyp at the gastroesophageal junction. Biopsy specimens were taken and histologic findings revealed a Barrett esophagus with high-grade intraepithelial neoplasia and local transition to a well-differentiated adenocarcinoma. According to the guidelines for esophageal cancer, an endoscopic mucosectomy was performed. Because a transmural peferation was suspected, the defect was closed with a few clips, and re-endoscopy showed welladapted wound edges. Histologic analysis revealed a well-differentiated adenocarcinoma (G1) emerging on the floor of a highly intraepithelial neoplasia of the Barrett 
mucosa, with minimal focal infiltration in the subepithelial connective tissue, no infiltration of lymphatics or vessels, and an incomplete resection by tumor infiltration of the lateral resection margin.

The case was discussed at an interdisciplinary tumor conference, and an oncologic resection was recommended. The patient was scheduled for a modified laparoscopic transhiatal extended resection of the cardia, with a reconstruction as RYGB as performed in bariatric surgery (Figure 1). An end-to-side esophagojejunostomy was performed with a circular stapler. No pouch was created. The initial postoperative course was uneventful. The final pathohistologic staging was (per TNM 2010): pT1b pN0 (0/11) M0, L0 V0 Pn0, R0, UICC-Stadium IA.

After 3 days, the patient developed a wound infection with a purulent secretion at one of the trocar incisions, accompanied by elevated infection parameters (white cell blood count [WBC] $11000 / \mathrm{mL}$, C-reactive protein [CRP] $214 \mathrm{mg} / \mathrm{L}$ ), which was treated conservatively. Because of the constantly increased inflammatory parameters (WBC $12300 / \mathrm{mL}$, CRP $242 \mathrm{mg} / \mathrm{L}$ ), chest radiography was performed and showed mild lower lobe infiltration. Drainage fluids were not suspicious, even after two routinely per-

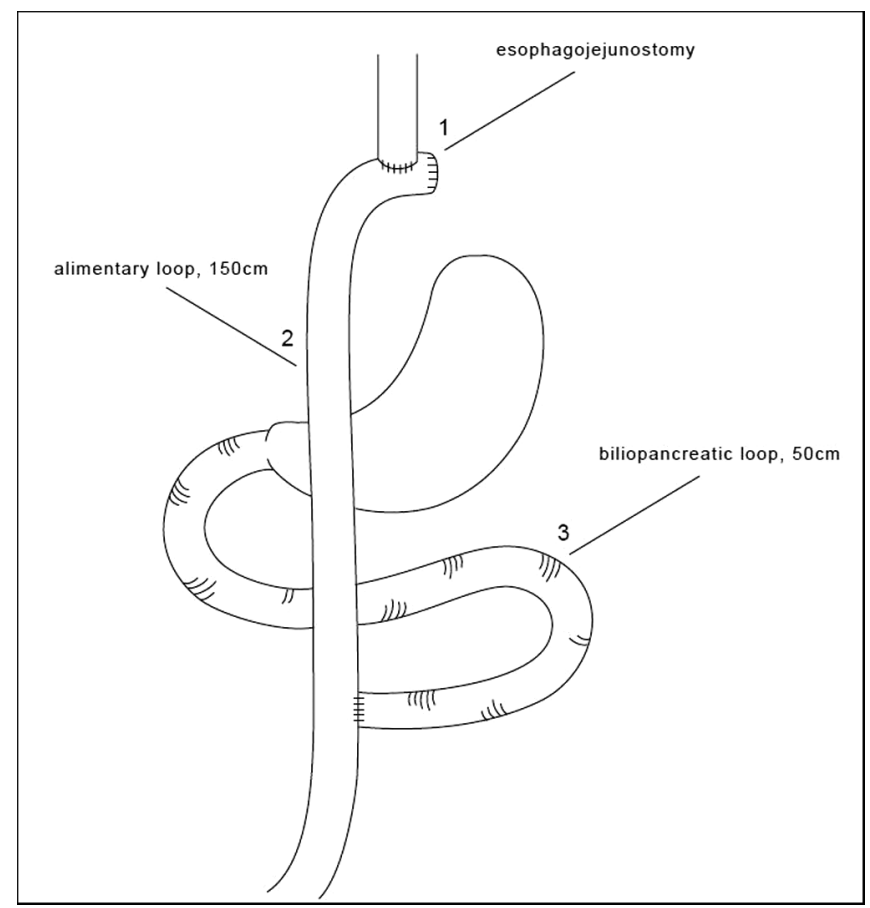

Figure 1. Modified laparoscopic transhiatal extended resection of the cardia (Merendino) with a reconstruction as RYGB. $1=$ the esophagojejunostomy, $2=$ the alimentary loop with a length of $150 \mathrm{~cm}, 3=$ the biliopancreatic loop with a length of $50 \mathrm{~cm}$. formed oral swallows of methylene blue dye. No satisfactory decrease in inflammatory markers was observed after initiating broad-spectrum antibiotic therapy. Radiography after a barium swallow was performed on postoperative day (POD) 6 without any evidence of an anastomotic leakage. One day later, abdominal computed tomography scan with contrast swallow was performed and no evidence of anastomotic leakage or another abdominal focus was noted. However, because inflammation parameters did not drop over time (WBC 23 400/mL, CRP $132 \mathrm{mg} / \mathrm{L}$ ), an additional computed tomography scan of the thorax and abdomen after oral contrast swallow was conducted after 11 days. It revealed an epiphrenic, proximal anastomotic leakage, with a small leakage into the right pleural space and pleural effusion with atelectasis of the right lower lobe and signs of stage 1 pleural empyema.

An urgent video-assisted thoracoscopy (VATS) was performed. Intraoperatively, nonpurulent, blood-tinged effusions were seen and removed, and two chest tubes were placed for sufficient drainage. In addition, intraoperative endoscopy was performed and a small esophagojejunal leakage was confirmed. It was sufficiently closed with an endoscopically applied Hem-o-lok clip the next day.

In the postoperative course, the underlying pleura drainage was purged 3 times daily with saline solution. Inflammatory markers decreased and the antibiotic therapy was discontinued. After 14 days, gastroscopy showed no evidence of ongoing insufficiency of the gastrojejunal anastomosis and oral food intake was begun. After negative microbiologic swabs were obtained, the pleural drainage tubes could be removed. The patient was discharged on POD 35 in good clinical condition.

The patient was seen in regular follow-up visits to our outpatient clinic. Eight months after surgery, his body weight had decreased to $114 \mathrm{~kg}$, corresponding to an excess weight loss of $66 \%$. Treatment for type 2 diabetes was no longer necessary and his dosage of antihypertensive medication was decreased. Vitamins, calcium, and magnesium were substituted as recommended.

\section{DISCUSSION}

We report the case of an obese patient who was initially scheduled for LRYGB, wherein at a preoperative gastroscopy, he was found incidentally to have a Barrett carcinoma. After incomplete mucosectomy, a modified oncologic resection was performed. Hospitalization was prolonged because of anastomotic leakage, which was sufficiently managed by VATS and endoscopic clipping. 
This case highlights a surgically modified procedure including both oncologic radicality and the weight loss effects of bariatric surgery. It also shows the complex pathway to diagnosis and treatment of possible complications after laparoscopic upper GI tumor and gastric bypass surgery. Advanced management of potential complications that can occur after gastric bypass surgery is highlighted. The greatest benefit for obese patients is to avoid open surgery.

A preoperative upper endoscopy is generally recommended to detect relevant GI pathologic conditions. This approach is supported by the results of De Moura Almeida et al, who demonstrated pathologic deviations in $77.2 \%$ of the endoscopies conducted preoperatively. ${ }^{4}$ Loewen et al screened 447 patients with a preoperative upper endoscopy. In 18\% of the patients, pathologic findings led to a change in medical treatment. ${ }^{5}$

The incidence of Barrett esophagus in obese patients is 3 times higher compared with the normal-weight population. Especially the combination of male sex, obesity, and GERD represents a high-risk constellation and is associated with a high risk for the development of adenocarcinoma of the esophagus. ${ }^{2}$

Once a patient is diagnosed, an appropriate treatment should be started. The aims of these treatments are improving GERD-related symptoms, including Barrett mucosa, and adequate weight reduction. Braghetto et al compared 3 different laparoscopic approaches in obese patients with GERD and Barrett esophagus: (1) calibrated fundoplication + posterior gastropexy; (2) fundoplication + vagotomy + distal gastrectomy + Roux-en-Y gastrojejunostomy; and (3) LRYGB. They found LRYGB the most effective treatment to improve GERD and to achieve weight loss. ${ }^{6}$ However, when histologic analysis reveals early Barrett carcinoma, endoscopic mucosectomy should be performed in the early stages according to recent guidelines. ${ }^{2}$ In the case of incomplete resection or advanced tumor growth, oncologic radicality should be the main priority.

Stein et al showed that in the case of T1a and T1b adenocarcinoma, a resection of the distal esophagus and esophagogastric junction with regional lymphadenectomy showed favorable results. It is a safe and feasible procedure associated with decreased morbidity and mortality. ${ }^{3}$ Therefore, to avoid open surgery and to maintain oncologic radicality combined with the weight-reducing effects of bariatric surgery, we performed the resection described above.

The incidence of intrathoracic anastomotic leakage after esophagectomy ranges from $2 \%$ to $19 \% .^{7}$ Diagnosis and management are complex and are currently the subject of controversial discourse. For diagnosis, direct visualization via endoscopy or radiologic imaging (eg, contrast swallow, computed tomography scan of the thorax and abdomen with oral contrast swallow) can be used.

Page et al saw high numbers of false-positive and falsenegative results after contrast swallow in their own study. ${ }^{8}$ Therefore, they performed a routine endoscopy 1 week after esophagectomy for anastomotic leakage detection. Their results showed that esophagoscopy is a safe and highly accurate method of diagnosing even very small leaks, and it simultaneously allows endoscopic treatment.

Since repeated blue and contrast swallows showed negative results and the drainage tubes were still in place, we performed not an early primarily endoscopy, what is otherwise recommended.? Consequently, the anastomotic leakage in our case was detected later, and an earlier endoscopy may have detected it earlier.

Because of the generally poor patient outcome after conservative treatments, reintervention (eg, metal or plastic stent, fibrin glue, endoscopic clipping, endoscopic vacuum-assisted closure systems) should be considered. ${ }^{10}$ In our case, leakage appeared to be $<10 \%$ of the circumference. Because of increased inflammation parameters and extended pleural effusion, a surgical approach was preferred in our case. In combination with endoscopic clipping, VATS allowed precise placement of the pleural drainage tubes. According to the current literature, endoscopic clip placement appears to be a suitable approach for smaller insufficiencies of the esophagus. ${ }^{11}$

The excess weight loss of $66 \%$ is at the upper end of the expected range. ${ }^{12}$

Obese patients are predisposed for severe Barrett esophagus and Barrett carcinoma. Therefore, routine preoperative endoscopy of the upper GI tract is highly recommended. Laparoscopic oncologic management of incidental early Barrett carcinoma is applicable in combination with routine bariatric surgery. VATS in combination with endoscopic clipping appears to be a sufficient treatment of small anastomotic leakages.

\section{References:}

1. Obeid A, Long J, Kakade M, Clements RH, Stahl R, Grams J. Laparoscopic Roux-en-Y gastric bypass: long term clinical outcomes. Surg Endosc. 2012;26(12):3515-3520.

2. Wang KK, Sampliner RE. 2008 updated guidelines for the diagnosis, surveillance and therapy of Barrett's esophagus. Am J Gastroenterol. 2008;103(3):788-797. 
3. Stein HJ, Feith M, Mueller J, Werner M, Siewert JR. Limited resection for early adenocarcinoma in Barrett's esophagus. Ann Surg. 2000;232(6):733-742.

4. de Moura Almeida A, Cotrim HP, Santos AS, et al. Preoperative upper gastrointestinal endoscopy in obese patients undergoing bariatric surgery: is it necessary? Surg Obes Relat Dis. 2008;4(2):144-1449, discussion 150-151.

5. Loewen M, Giovanni J, Barba C. Screening endoscopy before bariatric surgery: a series of 448 patients. Surg Obes Relat Dis. 2008;4(6):709-712.

6. Braghetto I, Korn O, Csendes A, Gutiérrez L, Valladares H, Chacon M. Laparoscopic treatment of obese patients with gastroesophageal reflux disease and Barrett's esophagus: a prospective study. Obes Surg. 2012;22(5):764-772.

7. Blencowe NS, Strong S, McNair AG, et al. Reporting of short-term clinical outcomes after esophagectomy: a systematic review. Ann Surg. 2012;255(4):658-666.
8. Page RD, Asmat A, McShane J, Russell GN, Pennefather SH Routine endoscopy to detect anastomotic leakage after esophagectomy. Ann Thorac Surg. 2013;95(1):292-298.

9. Siewert JR, Stein HJ, Bartels H. [Anastomotic leaks in the upper gastrointestinal tract]. Der Chirurg. 2004;75(11):10631070.

10. Hölscher AH, Fetzner UK, Bludau M, Leers J. [Complications and management of complications in oesophageal surgery]. Zentralblatt fur Chirurgie. 2011;136(3):213-223.

11. Schubert D, Pross M, Nestler G, et al. [Endoscopic treatment of mediastinal anastomotic leaks]. Zentralblatt fur Chirurgie. 2006;131(5):369-375.

12. O'Brien PE, MacDonald L, Anderson M, Brennan L, Brown WA. Long-term outcomes after bariatric surgery: fifteen-year follow-up of adjustable gastric banding and a systematic review of the bariatric surgical literature. Ann Surg. 2013;257(1): 87-94. 